\title{
Prioritized Data Calls with Time Threshold Performance Model in GSM
}

\author{
Kuboye B.M. \\ Computer Science Department, \\ Federal University of \\ Technology, \\ Akure, Ondo State \\ Nigeria \\ 1st author's email address
}

\author{
Alese B.K. \\ Computer Science Department, \\ Federal University of \\ Technology, \\ Akure, Ondo State \\ Nigeria \\ 3rd E-mail
}

\begin{abstract}
Global System for Mobile Communications (GSM) universal acceptability has led to the launching of lots of services ranges from telephony, Internet, multimedia, e-mails and congestion is now the resultant effect of this embracement. This work focuses on how the data calls can be managed on the network in order to minimized congestion experienced on the network. It classifies calls into different classes according to the type and nature of services offered. Thereafter, a level of priority was set among the classes so that the most important service will have access to the channel on the network by preempting the lower priority when there is congestion. The Markov chain's model is used to analyse the different classes of subscribers on the network and subsequently the steady state probabilities were derived. The blocking and dropping probabilities models for the different services were developed. The models were implemented to show how the congestion are minimised for different subscribers based on their priority levels. The impacts of priorities on different subscribers in the network were shown through the graphical display of the results.
\end{abstract}

\section{Keywords}

GSM, GPRS, EDGE, Real-time, Non-Real-time, Handoff

\section{INTRODUCTION}

Cellular telephone systems in the early 1980s were analog and therefore faced with the inability to handle the growing capacity needs in a cost-efficient manner. This problem occurs because the analog transceiver can only handle one call at a time. GSM is a digital technology family that overcomes the problem of analog systems. It handles more subscribers than the analog, offers high quality voice communication and low bandwidth $(96 \mathrm{~kb} / \mathrm{sec})$ data connections for fax and Short Message Service (SMS) [1]. GSM allow maximum freedom when making call, its voice communication is clear, service tariff is low and has a lot of added services like e-mail, browsing and so on. As a result of the success of voice communication on GSM, the demand for data connection services like browsing, videos, multimedia, and e-mail demands on mobile telephone have increased tremendously.

In the quest to meet this demand, 2.5G GSM was introduced through General Packet Radio Service (GPRS), High Speed Circuit-Switched Data (HSCSD) to give support for these demands. The continuing demand of accessing data on the mobile cellular radio started with the introduction of IP services in $2.5 \mathrm{G}$ and continues in $3 \mathrm{G}$ and $3.5 \mathrm{G}$. The $4 \mathrm{G}$ is considered to be the integration of the existing cellular networks and wireless LANs with added personalized mobile networks and broadband radio access networks to provide end-to-end IP connectivity. The aforesaid opens the relationship and connectivity that exist between the GSM technology and other higher technologies. All other technologies are based on the GSM technology, in other words, GSM technology stands as a backbone for other cellular technology. The ITU always puts the backward compatibility to the GSM as one of the criteria for rolling out new technology [2].

Traditionally, call demand type is either fresh or handover. Fresh call is a type of call that is demanding allocation of channel for communication for the first time Handover occurs when the mobile telephone network automatically transfers a call from one radio channel to another radio channel as mobile crosses adjacent cells. The means of allocating the scarce channels to calls to minimize congestion is an issue on the overall performance of the GSM networks. Several papers have been presented on the issue of channel allocation and three principal categories have been identified and they are; fixed channels allocation (FCA), dynamic channel allocation (DCA) and hybrid Channel Allocation (HCA) [3]. In FCA, a set of channels is permanently allocated to each cell based on pre-estimated traffic intensity. When a user requests for a channel for communication, it searches the free channel in its own cell, if there is free channel, the communication is granted, otherwise, is blocked. DCA allows dynamic allocation of channels as new calls arrive in the system. The entire sets of free channels are kept in a central pool and they are accessible to all the cells. As soon as a call finishes using a channel, the channel is automatically returned to a central pool where it can be accessible to all the cells in the base station. In HCA, few channels are allocated permanently to each cell and the remaining ones are on call by call basis. In other words, HCA is an integration of FCA and DCA schemes.

As a result of introduction of data GSM and consequent upon its high demand, the channel allocation strategies are affected. New channel allocation strategies that will accommodate the data traffic on GSM network have to be implemented. In literatures, three main static resource sharing schemes can be identified and they are Complete Sharing (CS), Complete Partition (CP) and Partial Partition [5][6][7]. 
The Complete Sharing (CS) allows all radio channels to be accessible for both data and voice traffics Complete Partitioning (CP), all channels are partitioned into two sets and each type of traffic is allowed to use only its dedicated set strictly. Partial Partitioning (PP) is a hybrid scheme where CP and CS schemes are combined. A set of channels is shared between voice and data traffics, and the remaining channels are partitioned into two sets, each partition being reserved for strict usage of its dedicated type of traffic.

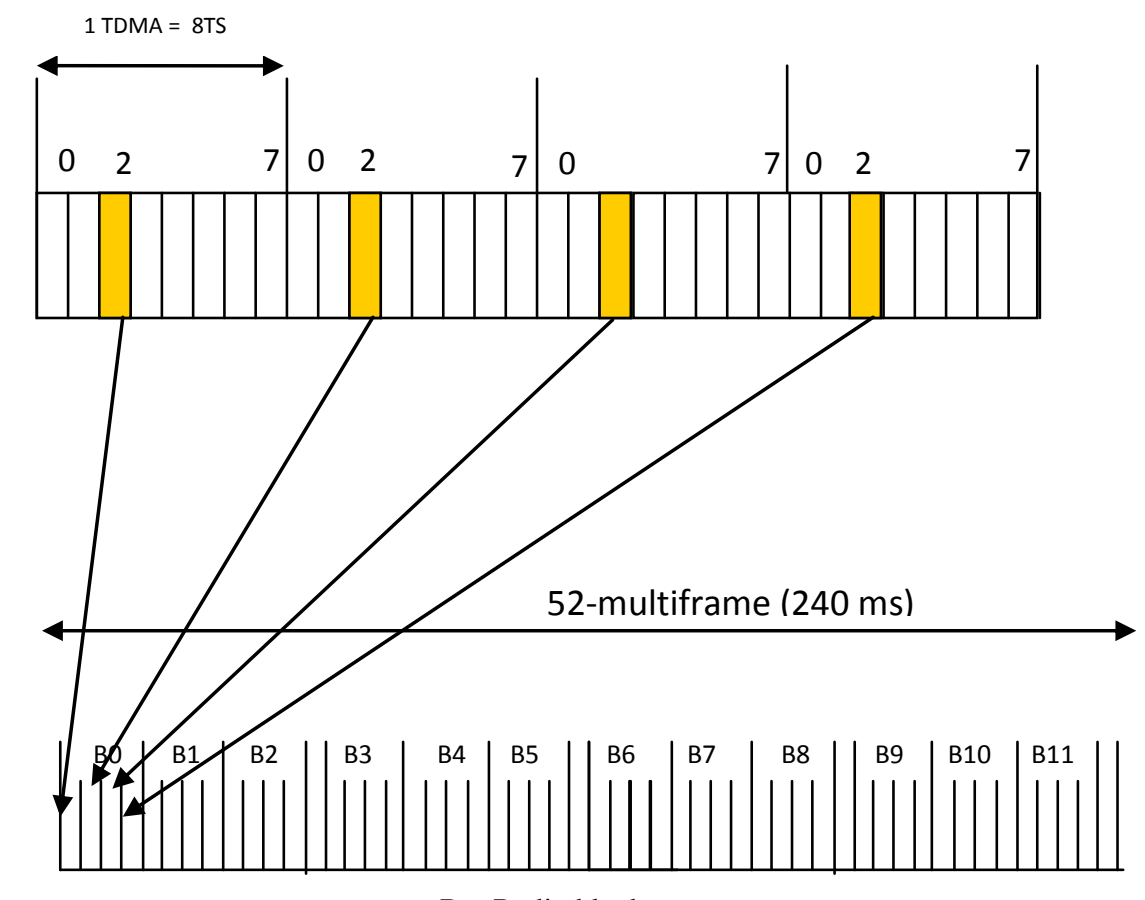

Bn: Radio block $n$

I: Idle frames

Fig 1. MultiFrame Structure (Source: [4])

\section{GPRS/EDGE MULTIPLE ACCESS CODING PRINCIPLES}

GSM uses a combination of Frequency Division Multiple Access (FDMA) and Time Division Multiple Access (TDMA). The FDMA scheme divides the GSM frequency band into a number of carrier frequencies, which in turn are splitted into timeslots by means of a TDMA scheme. A frame consists of a number of consecutive time-slots. The time-slots in a frame are then assigned to individual users. GPRS and EDGE data traffic uses the same radio interface as GSM voice calls hence radio resources available in the cell have to be shared among GSM and data traffic [7].

GPRS was conceived for the transfer of packets over a GSM infrastructure using packet data channels $(\mathrm{PDCH})$, with a simplified allocation of resources over the wireless link, and an IP transport among additional elements of the GSM network [4]. GPRS uses a 52-MultiFrame (MF) to differentiate it from GSM voice channels and multiplex multiple data packets from various sources. A PDCH simply corresponds to a timeslot (TS), while the 52-MF consists of 52 consecutive TDMA frames of the same TS and forms 4 idle frames and 12 RBs (B0 to B11). Each RB is composed of 4 TSs and is the basic unit in GPRS packet data transmission (see fig 1.0). For data traffic to cross the wireless link, IP packets are fragmented into radio blocks that are transmitted in 4 slots in identical positions within consecutive GSM frames over the same carrier frequency [8]. Most GPRS service provider allocates 3 TS per carrier frequency to carry GPRS packet data though subject to the preference of service provider. The total capacity $\mathrm{C}$ of the $3 \mathrm{TSs}$ can be calculated as: $(3 \mathrm{PDCH}) \mathrm{x}(12 \mathrm{RBs} / \mathrm{PDCH})=36 \mathrm{RBs}$. In GPRS, a Packet Control Unit (PCU) takes over all radio related control functions from the base station controller such as radio link control and medium access control. All RBs of a TS are assigned dynamically by the PCU (Packet Control Unit) and can be shared by various users with multiplexing technique [4].

GPRS/EDGE Mobile Station (MS) passes through three phases in transferring data: getting a Temporary Block Flow (TBF) assigned by the PCU, transmitting/receiving data, and returning the assigned TBF to the PCU. The TBFs, which are differentiated by TFI (Temporary Flow Identity), can be viewed as a physical connection between a MS and the underlying GPRS network. Since (E)GPRS traffics use the same radio interface as GSM calls, radio resources available in the cell have to be shared among GSM and (E)GPRS traffics. A GSM voice call needs the assignment of a single time-slot for its entire duration whereas in the (E) GPRS case, each time-slot can be shared among several users by assigning different Temporary Flow Identities (TFI) to the mobiles. Up to 32 TFI's can be allocated per TDMA. By monitoring the TFI of each radio block, a mobile can identify its own blocks and decode [1]). In addition to time-slot partitioning, (E)GPRS allows time-slot aggregation: depending on its 
Table 1.0: GPRS CODING (Source: [7][8])

\begin{tabular}{|l|l|l|l|l|}
\hline $\begin{array}{l}\text { GPRS Coding } \\
\text { Scheme }\end{array}$ & CS-1 & CS-2 & CS-3 & CS-4 \\
\hline $\begin{array}{l}\text { RLC block radio } \\
\text { (bytes) }\end{array}$ & 23 & 33 & 39 & 53 \\
\hline Data rate & 9.05 & 13.4 & 15.6 & 21.4 \\
\hline
\end{tabular}

Table 2.0: EDGE CODING (Source: [8])

\begin{tabular}{|l|l|l|l|l|l|l|l|l|l|}
\hline $\begin{array}{l}\text { GPRS Coding } \\
\text { Scheme }\end{array}$ & MC-1 & MC-2 & MC-3 & MC-4 & MC-5 & MC-6 & MC-7 & MC-8 & MC-9 \\
\hline $\begin{array}{l}\text { RLC radio block } \\
\text { (bytes) }\end{array}$ & 22 & 28 & 37 & 44 & 56 & 74 & 112 & 136 & 148 \\
\hline
\end{tabular}

(E)GPRS radio part is connected to the core network of GSM/EDGE by the Gb interface linking the Serving GPRS Support Node (SGSN) that performs mobility and subscriber management to the PCU that manages radio signals for packet traffic [4]. In the downlink, IP packets are fragmented and encapsulated into logical link control (LLC) frames by the SGSN. LLC frames are fragmented into smallerradio link control / media access control (RLC/MAC) blocks which add radio error protection bits to the data payload. The payload size of each radio block depends on the coding scheme. The GPRS standard defines 4 Coding Schemes (CS) namely CS1CS4 while EDGE allows 9 modulation and coding scheme (MC) which are MC1-MC9. Table 1.0 and 2.0 showed the comprehensive standard coding scheme specifications for GPRS and EDGE respectively. The tables show the RLC radio block sizes for GPRS and EDGE which defines the minimum data bytes that can be transferred over one PDCH time-slot. EDGE stands as a GPRS enhancement with improved radio modulation to allow higher bit rates.

\section{DATA TRAFIC MODEL}

In this work, voice handoff traffic, handoff data traffic, realtime (RT) and non-real-time (NRT) data traffic are considered. The data traffic occupies pool 2 (q2)and pool 3 (q3) (see fig 2.). Pool 2 will be shared between the handoff voice traffic and handoff data traffic. The complete sharing strategy has been proved that it has a better system utilization among the other channel allocation strategies [4]. Therefore, the complete sharing strategy is adopted in each partition.

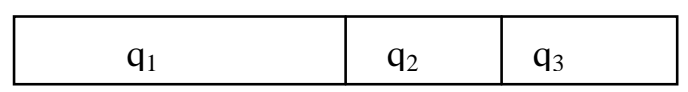

Fig 2. Complete sharing strategy
To guarantee the handoff voice performance, it was assumed that handoff voice traffic has preemptive priority over handoff data traffic in pool 2. Handoff voice calls is given a preemptive priority over handoff data calls because data can usually tolerate some degree of service degradation while voice is more delay sensitive [9]. When there are no channels available upon a handoff voice (hv) arrival, one of the handoff data packets in service is preempted. Likewise, to guarantee the performance of handoff data traffic (hd), when there are no channels available upon a handoff data (hd) arrival in pool 2 and pool 3, one of the NRT data packets in service in pool 3 will be preempted since hd has preemptive power over NRT and RT in pool 3. Also, RT has power to preempt non-realtime (NRT) data traffic in pool 3 if on arrival it finds no channel available for communication. When NRT calls arrives in pool 3 and no free channels available, then it will be blocked. Note that hd will be dropped if on arrival there is no RT and NRT in service and the maximum threshold to communicate with the former base station has elapsed, in the same way RT will be blocked if there is no NRT in service.

\section{THE TIME-THRESHOLD SCHEME (TTS)}

Guard channel scheme (GCS) gives priority to handoff calls for both data and voice, by reserving some channels for these handoff calls. GCS achieves low dropping probability, compared to blocking probability of new calls but at the cost of degradation in channel utilization. However, according to the complete sharing scheme (CSS) all available channels in a cell are shared by handoff and new calls. Therefore, CSS scheme minimizes the new call blocking probability and maximizes channel utilization. However, it is difficult to guarantee the required dropping probability of handoff calls [10]. Generally, GCS scheme is preferred by users since it decreases dropping probability (Pd), and CSS scheme is 
preferred by service providers since it maximizes system utilization.

Most of the studies have focused on prioritizing handoff calls at the expense of blocking new calls [9][11][12]. The common claim is that forced termination of an ongoing call is more irritating than blocking of newly originating calls. According to Candan and Salamah [10], this is true to some extent, but the level of the irritation depends on the elapsed real time of the ongoing call. For example, dropping an ongoing voice call is very irritating if it does not last for a moderate duration, whereas it is not that much annoying if it is approaching its end. Also, priority is usually associated with pricing; therefore normal conversations which are not so critical may tolerate handoff dropping at a lower price.

Considering the above arguments, a novel bandwidth allocation scheme for data calls which is based on fairness among calls was introduced and this outperforms the GCS and CSS schemes [9]. The main idea of this time-threshold scheme (TTS) is based on monitoring the elapsed real time of data calls and according to a time threshold parameter te, a handoff call is either prioritized or treated as a new call. Here in this work, te will be applied to handoff data call. Also, GCS scheme will be used to give handoff a level priority above real-time and non-real-time.

\section{DERIVATION OF PERFORMANCE MEASURES}

The performance analysis of radio resource allocation for multimedia traffic in this thesis is based on a 4-D Markov chain analysis model. The state $(\mathrm{i}, \mathrm{j}, \mathrm{k}, \mathrm{l})$ denote that there are $\mathrm{i}$ handoff voice calls, $\mathrm{j}$ handoff data packets, $\mathrm{k}$ RT data packets and 1 NRT data packets in the system. denotes the probability of the system in state $(\mathrm{i}, \mathrm{j}, \mathrm{k}, \mathrm{l})$. The total number of channels in the system is $\mathrm{C}$ and the number of reserved channels for handoff traffic is q2 while number of channels reserved for pool 3 traffic is q3. The arrival of voice call request forms Poisson process with a rate of

\subsection{Arrival time}

The arrivals of HD, RT and NRT data packets are assumed to be Poisson processes with rates $\lambda_{h d} \lambda_{r t}$ and $\lambda_{n r t}$, respectively, and $\lambda_{d}=\lambda_{h d}+\lambda_{r t}+\lambda_{n r t}$ is the aggregate data arrival rate. Assuming that $\lambda_{s}^{o}$ is the mean arrival rate of new call for service type $\mathrm{s}$ of one user, it follows that $\lambda_{h d}^{o}, \lambda_{r t}^{o}$ and $\lambda_{n r t}^{o}$ are the mean arrival rates of new call for service type HD, RT and NRT of one user of those classes respectively. The handoff data arrival rate depends on the number of active users $\left(\mathrm{x}_{\mathrm{s}}\right)$ in the system since if there is no user there cannot be any handoff. So it is given as $\lambda_{h d}=x_{s} \lambda_{h}^{o}$.

As explained earlier, a handoff data (hd) calls are controlled according to their threshold time (te). Recall that, the handoff data are accepted as long as the total number of Sv and hd are less than the number of available channels in $\mathrm{q} 2$ or the total number of hd in q3, otherwise, it blocked. However, in other to prevent some handoff to occupy the bandwidth unnecessarily, te is applied. A handoff data with te are accepted but subject to the fact that the te has not expired. Since te affects the prioritization of handoff calls, that is, as te increases, the number of prioritized calls decreases, $\beta$ implicitly affects the arrival rate of prioritized handoff calls [10]. Then, the arrival rate of prioritized handoff calls $\lambda_{h d}^{\prime}$ is directly proportional to both $\beta$ and the arrival rate of handoff calls, $\lambda$ hd. Therefore, $\lambda_{h d}^{\prime}$ can be written as

$\lambda_{h d}^{\prime}=\beta \cdot \lambda_{h d}$

Let $\beta$ be defined as the percentage of the prioritized handoff with the following heuristic formula (11),

$\beta=1 / \mu_{h d}-t_{e} / 1 / \mu_{h d}$

\subsection{Service}

\section{holding time}

The service time of $1 / \mu_{v}$ voice calls is assumed to be exponentially distributed with a mean of $1 / \mu_{0}$.The service holding time of HD, RT and NRT $1 / \mu_{v} \quad$ data packets is exponentially distributed with a $1 / \mu_{h d} \quad 1 / \mu_{r t} \quad 1 / \mu_{n r t}$ mean of and respectively. The aggregate

mean service rate is . It has been shown that it is reasonable to assume that the service of most data are

$$
\frac{1}{\mu_{d}}=\frac{1}{\mu_{h d}}+\frac{1}{\mu_{r t}}+\frac{1}{\mu_{n r t}}
$$

exponentially distributed, for some that are not exponentially distributed, they can be mounted on an exponentially distributed service rate that depends on state $\mathrm{x}$ through a bit rate $b_{s}(x)$, of this service [14]. According to the model, the service rate of type $\mathrm{s}$ that are exponentially distributed was given as $\mu_{s}(x)=x_{s} \mu_{s}^{o}$ while the one that will be mounted on an exponentially distributed service due to low holding time was given as $\mu_{s}(x)=x_{s} \frac{b_{s}(x)}{m_{s o}}, \mathrm{~m}_{\mathrm{so}}$ is the mean message size of type $\mathrm{s}$ calls. For simplicity, in this model, the second assumption is adopted since all data that are not exponentially distributed can be mounted on exponentially distributed service. Therefore, service rate of HD, RT and NRT are

$$
\mu_{h d}=j_{h d} \frac{b_{h d}}{m_{h d}}, \quad \mu_{r t}=k_{r t} \frac{b_{r t}}{m_{r t}} \text { and } \quad \mu_{n r t}=l_{n r t} \frac{b_{n r t}}{m_{n r t}}
$$

respectively.

\subsection{The Underlying Markov Chain}

The proposed scheme here can be modeled with a 4-D Markov chain as shown in Fig. 3.

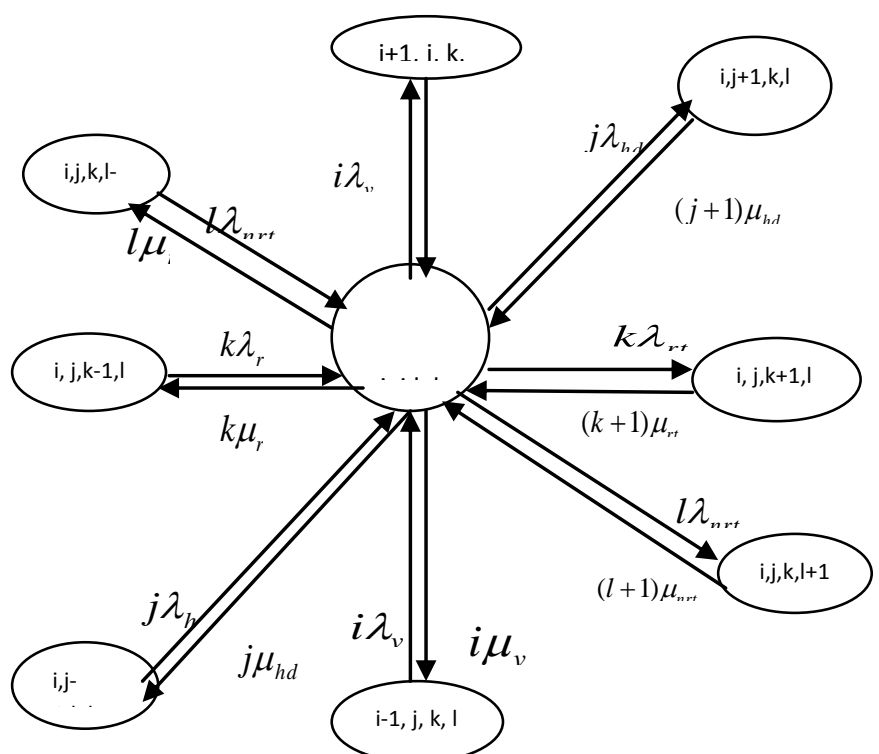

Fig 3. Four State Markov Chain for Data Analysis 
The state space of the Markov chain is

$P(i: j: k: l)=\lim _{t \rightarrow \infty} P\left(\left(q_{2}(t)=i\right),\left(q_{2}+q_{3}\right)(t)=j, q_{3}(t)=k, q_{3}(t)=l\right)$

Let $\mathrm{S}$ be the set of feasible states,

$S=\left\{(i, j, k, l) \mid 0 \leq i \leq q_{2}, 0 \leq j \leq q_{2}+q_{3}, 0 \leq k \leq q_{3}, 0 \leq l \leq q_{3}\right\}$

By applying the constraint to the set of $\sum_{s} \pi_{i, j, k, l}=1$ to the set of balance equations, we can obtain the steady-state Probability $\pi_{i, j, k, l}$ to evaluate the performance metrics of the system. The steady state probability is calculated as follows:

$\pi_{i, j, k, l}=\frac{1}{P(0,0,0,0)} \cdot\left(\frac{\ell_{v}^{i}}{i !} \cdot \frac{\ell_{h d}^{j}}{j !} \cdot \frac{\ell_{r t}^{k}}{k !} \cdot \frac{\ell_{n r t}^{l}}{l !}\right)$

where $\mathrm{P}(0,0,0,0,0)$ is the steady-state probability of the system being idle

From the normalization equation, $\sum_{s} \pi_{i, j, k, l}=1$ we obtain

$P(0,0,0,0)=\sum_{a=0}^{C-q_{1}} \frac{\left(\ell_{v}+\ell_{n d}+\ell_{r t}+\ell_{n r t}\right)^{a}}{a !}$

where $\mathrm{a}=\mathrm{i}+\mathrm{j}+\mathrm{k}+1$ and $\rho=\frac{\lambda}{\mu}$. Hence we obtain the state probability as

$\pi_{i, j, k, l}=\left(\frac{\ell_{v}^{i}}{i !} \cdot \frac{\ell_{h d}^{j}}{j !} \cdot \frac{\ell_{n}^{k}}{k !} \cdot \frac{\ell_{n n}^{l}}{l !}\right) / \sum_{a=0}^{c-q_{1}}\left(\ell_{v}+\ell_{h d}+\ell_{r t}+\ell_{n r t}\right)^{a} / a !$

Using the state probability, the following performance metrics can be derived

i Handoff Data Packet dropping: A handoff data call is blocked when upon arrival, q2 is filled up with $\mathrm{Sv}$ and Hd and $\mathrm{q} 3$ is filled up with $\mathrm{Hd}$. The packet blocking probability of HD data traffic, Prtb, is expressed as

$P_{h d b}=\sum_{i=0}^{q_{2}} \sum_{j=0}^{q_{2}-i+q_{3}} \sum_{k=0}^{q_{3}} \sum_{l=0}^{q_{3}} \pi_{i, j, k, l}$

ii A Real-time data call is blocked when upon arrival; q3 is filled up with Hd and RT. The packet loss probability of RT data traffic, Prtb, is expressed as

$$
P_{r t b}=\sum_{i=0}^{q_{2}} \sum_{j=0}^{q_{2}+q_{3}} \sum_{k=0}^{q_{3}-j} \sum_{l=0}^{q_{3}} \pi_{i, j, k, l}
$$

iii The packet blocking probability of NRT traffic,

$$
P_{n r t b}=\sum_{i=0}^{q_{2}} \sum_{j=0}^{q_{2}+q_{3}} \sum_{k=0}^{q_{3}} \sum_{l=0}^{q_{3}-(j+k)} \pi_{i, j, k, l}
$$

\section{ANALYTICAL SIMULATION AND PERFORMANCE RESULTS FOR DATA COMMUNICATION MODEL}

In this paper, the parameters used in the analytical simulation are listed in the table 3.

\begin{tabular}{|c|c|}
\hline $\begin{array}{l}\text { Total channel in the } \\
\text { cell }\end{array}$ & 22 \\
\hline Total channel for q2 & 6 \\
\hline Total channel for $\mathrm{q} 3$ & 5 \\
\hline Service rate $1 / \mu_{d}$ & $600 \mathrm{~s}, 300 \mathrm{~s}$ \\
\hline $\begin{array}{l}\text { Arrival rate for voice } \\
\lambda_{v}\end{array}$ & $\begin{array}{l}1.67-5.0 \mathrm{call} / \mathrm{s} \approx 100- \\
250 \text { calls }\end{array}$ \\
\hline $\begin{array}{l}\text { Arrival rate for handoff } \\
\text { data } \lambda_{h}\end{array}$ & $\begin{array}{l}2.5-5.83 \mathrm{call} / \mathrm{s} \approx 150-250 \\
\text { calls }\end{array}$ \\
\hline $\begin{array}{l}\text { Arrival rate for } \\
\text { Realtime data } \lambda_{r}\end{array}$ & $\begin{array}{l}3.33-6.67 \mathrm{call} / \mathrm{s} \approx 200-300 \\
\text { calls }\end{array}$ \\
\hline $\begin{array}{l}\text { Arrival rate for Non- } \\
\text { realtime data } \lambda_{n}\end{array}$ & $\begin{array}{l}5-8.33 \mathrm{call} / \mathrm{s} \approx 300-500 \\
\text { calls }\end{array}$ \\
\hline $\begin{array}{l}\text { Offerred load in erlangs } \\
\text { for special } \rho_{\mathrm{s}}\end{array}$ & $5.0-15,3.3-10$ \\
\hline $\begin{array}{l}\text { Offerred load in erlangs } \\
\text { for special } \rho_{\mathrm{h}}\end{array}$ & $7.5-17.5,5-11.7$ \\
\hline $\begin{array}{l}\text { Offerred load in erlangs } \\
\text { for special } \rho_{\mathrm{r}}\end{array}$ & $10-20,6.7-13.3$ \\
\hline $\begin{array}{l}\text { Offerred load in erlangs } \\
\text { for special } \rho_{\mathrm{n}}\end{array}$ & $15-25,2.5-9.2$ \\
\hline $\mathrm{P}_{\mathrm{v}}$ & $\begin{array}{l}\text { Blocking Probability of } \\
\text { voice call }\end{array}$ \\
\hline $\mathrm{P}_{\mathrm{h}}$ & $\begin{array}{l}\text { Dropping Probability of } \\
\text { handoff data (HD) call }\end{array}$ \\
\hline$\overline{P_{r t}}$ & $\begin{array}{l}\text { Blocking Probability of } \\
\text { realtime (RT) data call }\end{array}$ \\
\hline$\overline{P_{\text {nrt }}}$ & $\begin{array}{l}\text { Blocking Probability of non } \\
\text { realtime (NRT) data call }\end{array}$ \\
\hline
\end{tabular}

Table 3. Data Analytical Simulation Parameters 
The blocking probabilities of Data traffic for all data types in the presence of voice call when simulation was done with service time of 600s and 300s are shown Fig. 4. The blocking probability under 600 s was higher than the one done with 300 s.

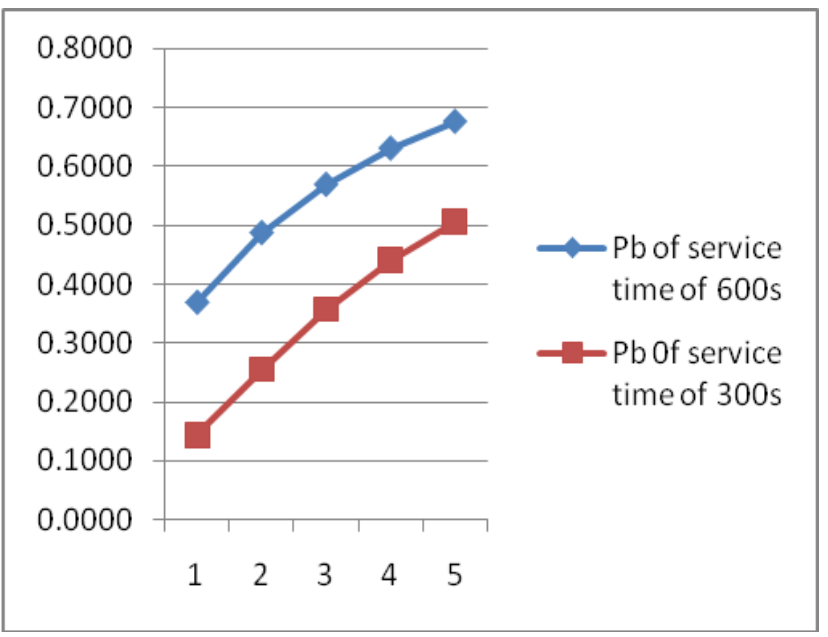

Fig 4. Graph representation of Data traffic probabilities in the presence of Voice call

The situation when time threshold (te) is applied to the HD is shown Fig 4, this further reduced the blocking probabilities. The reason for the slight reduction in the blocking probabilities is the presence of voice traffic and Handoff data traffic. The voice traffic will always have preference over the data traffic since data traffic can usually tolerate some degree of service [10]. Also, the threshold is only applied to the handoff data, therefore the effect of this is not too significant when compared to the overall probabilities.

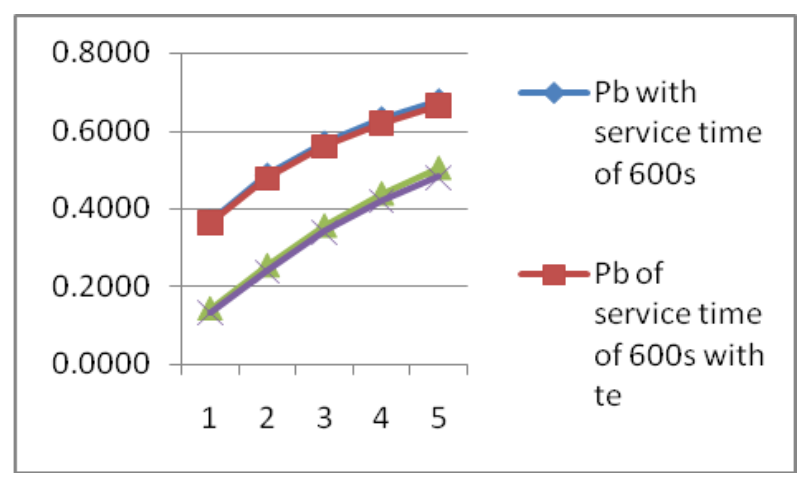

Fig 5. Graph representation of Data traffic probabilities in the presence of Voice call when te was applied

The blocking probabilities of data traffic when there is no voice traffic in $\mathrm{q} 2$ is shown inFig.6. These shows there are more channels available for the HD users in q2, this account for the further reduction of blocking probabilities of data traffic in the cell. There is further reduction in blocking probabilities when time threshold (te) was applied to the HD traffic though the te was only applied to HD and number of $\mathrm{HD}$ are always minimal.

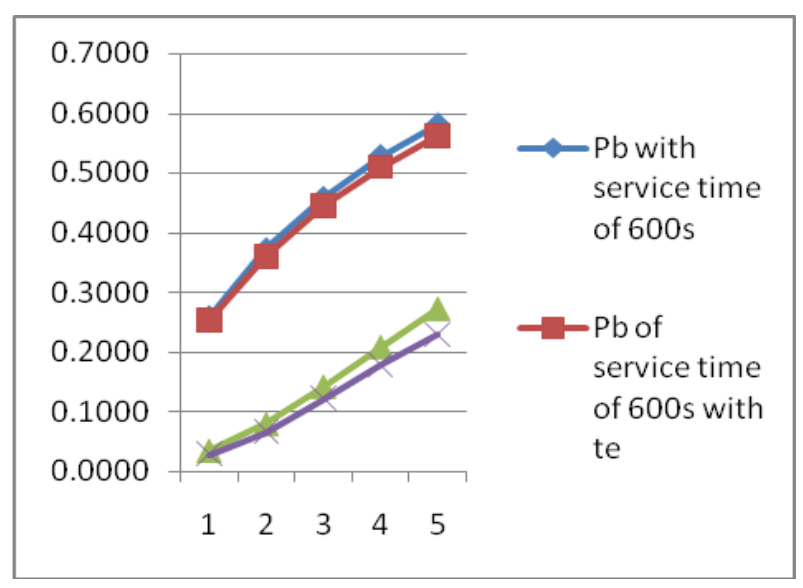

Fig 6. Data traffic without voice

Fig 7. show a situation when HD occupying $\mathrm{q} 2$ and RT occupy q3. Also, the effect of te is also shown. The least blocking probabilities are achieved when te was applied to the HD. Fig 8. show a situation when HD occupying q2 and NRT occupy q3. Also, the effect of te is also shown. The least blocking probabilities are achieved when te was applied to the HD.

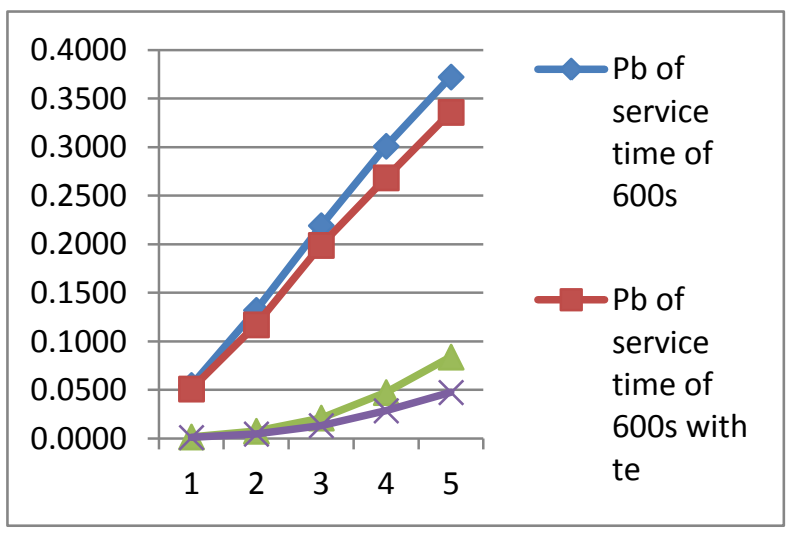

Fig 7. HD and RT

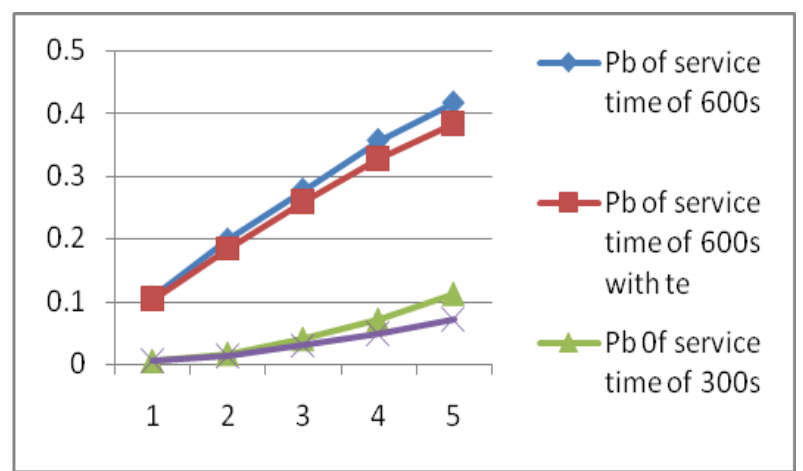

Fig 8.0 HD and NRT

\section{CONCLUSION}

This work focuses on how the congestion experienced on the GSM network can be minimized. It classifies subscribers into different classes according to the type and nature of services offered. Thereafter, a level of priority was set among the classes so that the most urgent and important service will have access to the channel on the network by preempting the lower priority when there is congestion and there is no free channel to communicate. Also, the voice communication and data 
communication over the GSM network using the different classes of subscribers were analyzed. The effects of each class on the network and its impact on another class are shown. In other to have a better service offerrings, a time threshold was applied to the handoff voice and data, thus, preventing these classes of calls from occuppying channels unneccessarily will give others chance to seize the channels. The results of this shows a significant reduction in the blocking probabilities of the RT and NRT calls.

\section{REFERENCES}

[1] Kuboye, B.M., (2006). Development of a Framework for Managing Congestion in Global System for Mobile Communications in Nigeria, unpublished M.Tech Project. F.U.T.A. Nigeria.

[2] Janeviski, T., (2003).Traffic Analysis and Design of Wireless IP Networks Artech House, INC ISBN 158053-331-0.

[3] Gupta and Sachan, (2007). Distributed Dynamic Channel Allocation Algorithm for Cellular Mobile Network, Journal of Theoretical and Applied Information Technology.

[4] Tsai, C., et al (2005) Synchronous and Multiplexed Time Slots and Radio Blocks Allocation for Mobile Packet Data Networks, Proceedings of the 19th International Conference on Advanced Information Networking and Applications, (Aina'05) IEEE.

[5] Dahmouni H. et al (2005). Performance Modeling of GSM/GPRS Cells with Different Radio Resource Allocation Strategies, Methods and Tools for Traffic Engineering, France Telecom R \& D, Belfort, France.

[5] Fang and Ghosal (2003). Performance Modeling and QoS Evaluation of MAC/RLC Layer in GSM/ GPRS Networks. Proceeding of IEEE ICC, Alaska, USA.
[6] Budura G. et al (2009). Traffic Models and Associated Parameters in SM/(E)GPRS Networks, WSEAS Transactions on Communications, Issue 8, Volume 8.

[6] Marsan M. A., (2001). Efficient Estimation of Call Blocking Probabilities in Cellular Mobile Telephony Networks with Customer Retrials, IEEE Journal on Selected.

[9] Tang L., Shensheng T., and Wei L.(2004). A Channel Allocation Model with Preemptive Priority for Integrated Voice/Data Mobile Networks, Proceedings of the First International Conference on Quality of Service in Heterogeneous Wired/Wireless Networks, (Qshine'04) 0-7695-2233-5/04 IEEE. 2004.

[10] Candan And Salamah. (2006). Performance and Analysis of a Time-Threshold Based Bandwidth Allocation Scheme for Data Calls in Cellular Networks, 0-76952699-3/06 (c) IEEE.

[11] Roszik J., Sztrik J., Che-Soong K. (2003). Retrial Queues in The Performance Modeling of Cellular Mobile Networks Using Mosel", International Journal of Simulation Vol. 6 No. 1-2.

[12] Chen and $\mathrm{Wu}$, (2005) Performance Analysis of Radio Resource Allocation for Multimedia Traffic in Cellular Networks, Proceedings of the 19th International Conference on Advanced Information Networking and Applications (Aina'05), IEEE.

[13] Begain K., et al.(2000) Analytical Call Level Model of GSM/GPRS Network", in Proceedings of Spects'00, SCS Symposium on Performance Evaluation of Computer and Telecommunication Systems, Vancouver, Canada 